\title{
SCALABLE RECOMMENDATION WITH SOCIAL CONTEXTUAL INFORMATION
}

\author{
Fabian A ${ }^{1}$, S.Lakshmi Sridevi ${ }^{2}$ \\ ${ }^{I}$ MCA, Department of Computer Applications, Hindustan University, Chennai, India \\ ${ }^{2}$ Assistant Professor, Department of Computer Applications, Hindustan University, Chennai, India
}

\begin{abstract}
Recommender systems are used to achieve effective and useful results in a social networks. The social recommendation will provide a social network structure but it is challenging to fuse social contextual factors which are derived from user's motivation of social behaviors into social recommendation. Here, we introduce two contextual factors in recommender systems which are used to adopt a useful results namely a) individual preference and $b$ ) interpersonal influence. Individual preference analyze the social interests of an item content with user's interest and adopt only users recommended results. Interpersonal influence is analyzing user-user interaction and their specific social relations. Beyond this, we propose a novel probabilistic matrix factorization method to fuse them in a latent space. The scalable algorithm provides a useful results by analyzing the ranking probability of each user social contextual information and also incrementally process the contextual data in large datasets.
\end{abstract}

Keywords: social recommendation, individual preference, interpersonal influence, matrix factorization.

$* * *$

\section{INTRODUCTION}

Social network users generate large volumes of information, which makes it necessary to exploit highly accurate recommender systems to assist them in finding useful results. Social contextual factors work on the contextual information and integrate them into a unified recommendation framework. The contextual factors such as individual preference and interpersonal influence work on the each users behaviors to adopt the recommended information. These two factors will highly provide an useruser interactions with their interpersonal influence or social relation which tells whether the user has close relation with item senders and individual preference which adopt the behavior of the user content whether the user likes the item or not. A novel probabilistic matrix factorization method is used in this social recommendation to fuse the user-user and user-item comparison and relations in a latent space. In order to analyze or operate the recommended contents we use effective scalable algorithm to find a probability of each user item content which shows a high valuable item content and it incrementally process the data.

\section{RELATED WORKS}

We review several major approaches to recommendation methods. Collaborative filtering and content based filtering have been widely used to help users find out the most valuable information. With the emerge of social networks, researchers design trust-based (Ester) [7] and influencebased (J. Huang, 2010) [6] methods to take use of the power coming from user relationships for recommendation. Storing context data using data cubes, called context cubes, is proposed in (L. D. Harvel, 2004) [14] for developing context-aware applications that use archive sensor data. In this work, data cubes are used to store historical context data and to extract interesting knowledge from large collections of context data.
(A. Karatzoglou, 2010) [4] Proposed a model based CF approach for making recommendation with respect to rich contextual information, namely multiverse recommendation. Specifically, they modeled the rich contextual information with item by Ndimensional tensor, and proposed a novel algorithm to make tensor factorization. (Soo, 2004) [11] Proposed a prototype design for building a personalized recommender system to recommend travel related information according to users' contextual information. (M.H. Park, 2007) [9] Proposed a location based personalized recommender system, which can reflect users' personal preferences by modeling user contextual information through Bayesian Networks.

(Hao Ma) [17] Analyse latent factor using probabilistic matrix factorization, we learn the user latent feature space and item latent feature space by employing a user social network and a user-item matrix simultaneously and seamlessly. (H. Ma, 2011) [22] Proposed a matrix factorization framework with social regularization. But this work only constrains user feature vectors from interpersonal side but ignores users' individual side, which makes the framework lack of complete contextual information to further improve the recommendation accuracy.

\section{PROPOSED SYSTEM}

To overcome from the drawback of the existing system we proposed ContextMF, a novel social recommendation model utilizing social contextual factors, individual preference and interpersonal influence. . This design following the three Primary goals:

1) Reduced bandwidth server cost due to forwarding a questions in a selected people in their friend list.

2) Quick response due to low number of overhead nodes. 
3) Large datasets can be adopted based on user's behaviors.

4) Finding the probability of each item content by matching with users interests.

\subsection{System Architecture}

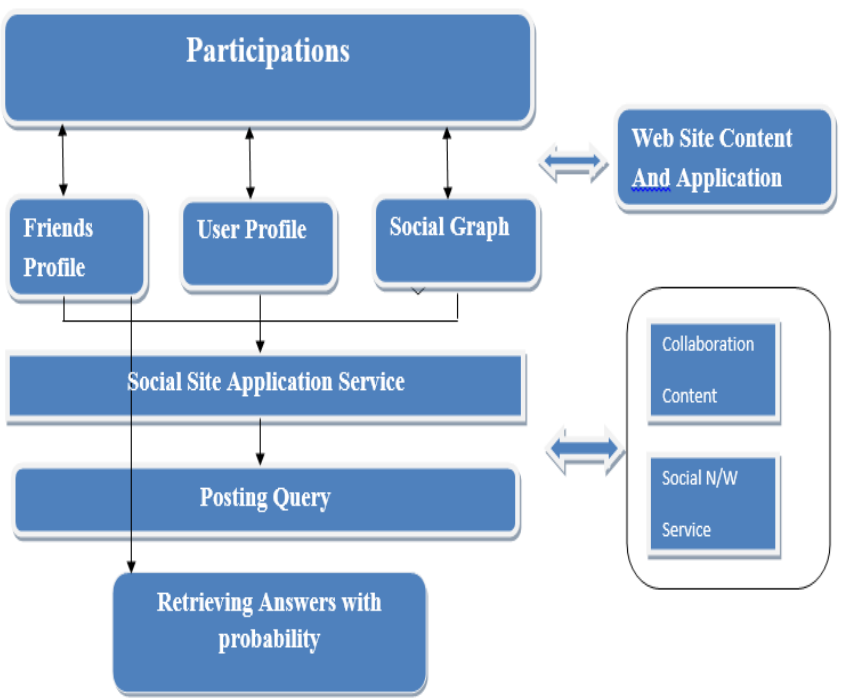

Fig 1: Proposed architecture

\section{MODULE}

\subsection{Authentication}

Authentication is a process to check whether the right person is getting into the profile or not. If the person ID and password does not match means an error message will be displayed. Here the Authentication process is use to checked for all user and admin of the page. After the admin authenticated he can get into his profile and do some modification which are viewed by his friends.

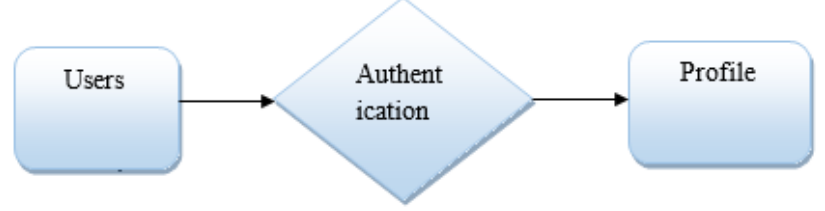

Fig 2: Authentication

\subsection{Managing Profile}

After the authentication the users are getting into their profile. The users can change their account settings. Here they can change their old password, name, profile picture etc. These process are maintained by the users only

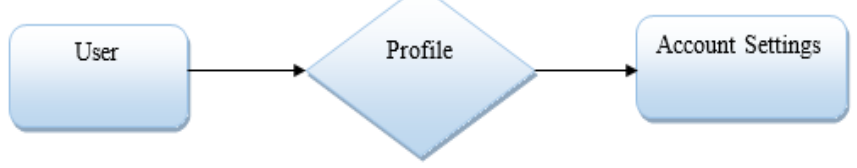

Fig 3: Managing Profile

\subsection{Invite Friends}

Here the users can invite or give friend request to their known persons. By giving the request they will be added into their friends list. If the users no need their friendship means they can delete the friend details.

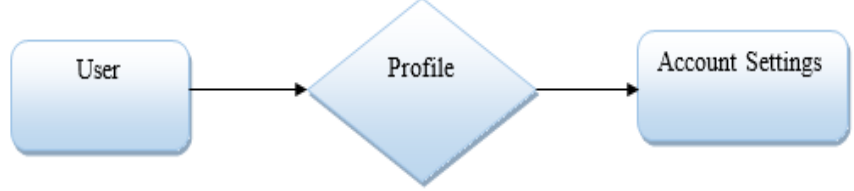

Fig 4: Invite friends

\subsection{Posting Query}

Posting Query is a module in which the users are used to post the query like sharing. If friends may know the answer means they will post some answer. The query can be viewed only if they both are friends. Else they can't view the query.

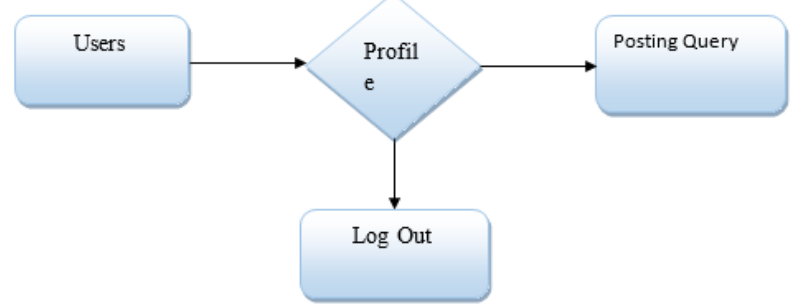

Fig 5: Posting query

\subsection{Answer Analysis}

After posting the query the friends will give some answers. That answers are compared by using Scalable algorithm. The main concept of this algorithm is user to compare the answers and provide the correspondent answer to the question.

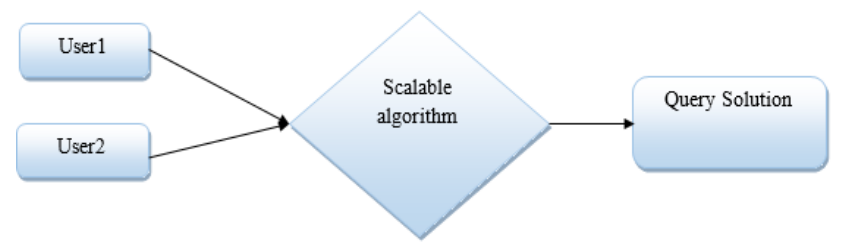

Fig 6: Answer analysis

\subsection{Retrieval Answers with its Probability}

This module is used to give answer to the posted query user. The answers are analysed with scalable algorithm to get the probability of a answer by matching user intrests and has been posted. The final answer is viewed by the user who has been posted some query.

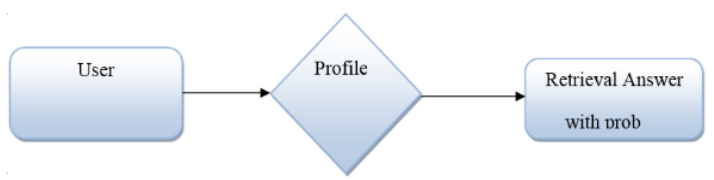

Fig 7: Retrieval answer prob 


\section{TECHNIQUE}

\subsection{Scalable Algorithm}

We further provide a scalable algorithm which can incrementally process the data. We conduct experiments on both Facebook style bidirectional and Twitter style unidirectional social network datasets. The empirical results and analysis on these two large datasets demonstrate that our method significantly outperforms the existing approaches. We provide a scalable algorithm to incrementally process the data so that it can achieve the scalable recommendation goal and be used on large real applications. The time cost is linear to the size of recommended items and users. This algorithm is used for achieving these goal:

1. Reduced bandwidth server cost due to forwarding a questions in an selected people in their Friend list.

2. Quick response due to low number of overhead nodes.

3. It can incrementally process the large sets of data.

\section{CONCLUSION AND FUTHRE \\ ENHANCEMENT}

We proposed a novel social recommendation model utilizing social contextual factors, i.e., individual preference and interpersonal influence. We conducted extensive experiments on two large real world social network datasets, and showed that social contextual information can greatly boost the performance of recommendation on these social network data and Also, the proposed algorithm is general and can be easily adapted according to different real-world recommendation scenarios.

In our future work we primarily add the review of the interest on the basis of highly recommended query. The value which will be most near to query will be shown first in the comment list.

\section{ACKNOWLEDGEMENTS}

It's a matter of pride and privilege for me to express my deep gratitude to the management of HITS for providing me the necessary facilities and support.

\section{REFERENCE}

[1]. G. Adomavicius, A. Tuzhilin, "Toward the next generation of recommender systems: a survey of the stateof-the-art and possible extensions," in TKDE, vol.17, no.6, pp.734-749, 2005.

[2]. H. Ma, H. Yang, M.R. Lyu, and I. King,"SoRec: Social Recommendation Using Probabilistic Matrix Factorization," in CIKM'08.

[3]. H. Ma, D. Zhou, C. Liu, M.R. Lyu, and I. King, "Recommender System with Social Regularization," in WSDM'11, pp. 287-296.

[4]. A. Karatzoglou, X. Amatriain, L. Baltrunas, and N. Oliver. Multiverse recommendation: n-dimensional tensor factorization for contextaware collaborative filtering. In RecSys '10, pages 79-86, 2010.
[5]. R. Bond and P.B. Smith, "Culture and Conformity: a Meta-analysis of Studies Using Asch's (1952b, 1956) Line Judgment Task," Psychological Bulletin, vol.119, no.1, pp. 111-137,1996.

[6]. J. Huang, X. Cheng, J. Guo, H. Shen, and K. Yang. Social recommendation with interpersonal influence.In Proceedings of the 19th European Conference on Artificial Intelligence (ECAI), pages 601-606, 2010.

[7]. M. Jamali and M. Ester. Trustwalker: a random walk model for combining trust-based and item-based recommendation. In Proceedings of the 15th ACM SIGKDD International Conference on Knowledge

Disco

[8]. M.J. Salganik, "Experimental Study of Inequality and Unpredictability in an Artificial Cultural Market," Science, vol.311, pp. 854-856, 2006.

[9]. M.-H. Park, J.-H. Hong, and S.-B. Cho. Location-based recommendation system using bayesian user's preference model in mobile devices. In UIC'07, pages 1130-1139, 2007

[10]. D.M. Blei, A.Y. Ng, and M.I. Jordan, ”Latent Dirichlet Allocation," JMLR, vol.3,pp. 993-1022,2003.

[11]. H.-W. Tung and V.-W. Soo. A personalized restaurant recommender agent for mobile e-service. In EEE'04, pages 259-262, 2004.

[12]. M. Balabanovi'c and Y. Shoham, "Fab: Content-based, Collaborative Recommendation," Commun. ACM, vol.40, no.3, pp. 66-72, 1997.

[13]. O. Phelan, K. McCarthy, and B. Smyth. "Using twitter to recommend real-time topical news," in RecSys'09, pp. 385-388, 2009.

[14]. L. D. Harvel, L. Liu, G. D. Abowd, Y.-X. Lim, C. Scheibe, C. Chatham, Context Cube: Flexible and Effective Manipulation of Sensed Context Data, in: Pervasive, 51-68, 2004.

[15]. K. Stefanidis, E. Pitoura, P. Vassiliadis, "Managing contextual preferences," in Info. Sys., vol.36, iss. 8, pp. 1158-1180, 2011.

[16]. H. Zhu, E. Chen, K. Yu, H. Cao, H. Xiong, and J. Tian, "Mining Personal Context-Aware Preferences for Mobile Users," in ICDM '12, pp. 1212-1217.

[17]. H. Ma, D. Zhou, C. Liu, M. R. Lyu, and I. King. Recommender system with social regularization. In Proceedings of the 4th ACM International Conference on Web Search and Data Mining (WSDM), pages 287-296, 2011.

[18]. Hao Ma, Haixuan Yang, Michael R. Lyu, Irwin King SoRec: Social Recommendation Using Probabilistic Matrix Factorization Dept. of Computer Science and Engineering The Chinese University of Hong Kong Shatin, N.T., Hong Kong \{hma, hxyang, lyu, king $\} @$ cse.cuhk.edu.hk 